\title{
Abstract: Self-Supervised 3D Context Feature Learning on Unlabeled Volume Data
}

\author{
Maximilian Blendowski, Mattias P. Heinrich \\ Institut für Medizinische Informatik, Universität zu Lübeck \\ blendowski@imi.uni-luebeck.de
}

Deep learning with convolutional networks (DCNN) has established itself as a powerful tool for a variety of medical imaging tasks. However, DCNNs in particular require strong monitoring by expert annotations, which cannot be generated cost-effectively by laymen. In contrast to manual annotations, the mere availability of medical volume data is not a problem. In our MICCAI 2019 conference paper [1], we present a fully self-supervised method to learn 3D context features by exploiting data-inherent patterns and leveraging anatomical information freely available from medical images themselves.

Closely related to the context prediction of neighboring patches as classification task (e.g. top/bottom, left/right) by Doersch et al. [2], we alter this self-supervised pretext loss to a more flexible regression task. We propose to appropriately leverage spatial information in 3D scans by predicting orthogonal offsets of two planar patches that are extracted with a small intermediate gap. In addition, we use an auxiliary decoder network for $2 \mathrm{D}$ heatmap regression that increases the robustness of this offset regression.

Using our Vantage Point Forest method [3] for an approximate k-Nearest Neighbor search in an atlas database, we predict labels without any subsequent finetuning strategies for a multi-organ segmentation task. Compared to a naive $3 \mathrm{D}$ extenstion of [2], we obtain a large increase in mean Dice scores from $55.2 \%$ to $65.5 \%$. While we only trained with spatial relations, we also achieve state-of-the-art results for one-shot-segmentation on a public abdominal CT dataset.

Acknowledgement. This work was supported by the German Research Foundation (DFG) under grant number 320997906 (HE 7364/2-1) and by the NVIDIA Corporation with their GPU donations. Find our code here:

https://github.com/multimodallearning/miccai19_self_supervision

\section{References}

1. Blendowski M, Nickisch H, Heinrich MP. How to learn from unlabeled volume data: self-supervised 3d context feature learning. In: MICCAI. Springer; 2019. p. 649-657.

2. Doersch C, Gupta A, Efros AA. Unsupervised visual representation learning by context prediction. In: Proc IEEE Int Conf Comput Vis; 2015. .

3. Heinrich MP, Blendowski M. Multi-organ segmentation using vantage point forests and binary context features. In: MICCAI. Springer; 2016. p. 598-606. 